\title{
Exploring the Impact of Emotion on Visual Judgement
}

\author{
Lane Harrison* \\ Remco Chang \\ Aidong Lu \\ UNC-Charlotte \\ Tufts University \\ UNC-Charlotte
}

\begin{abstract}
Existing research suggests that individual personality differences can influence performance with visualizations. In addition to stable traits such as locus of control, research in psychology has found that temporary changes in affect (emotion) can significantly impact individual performance on cognitive tasks. We examine the relationship between fundamental visual judgement tasks and affect through a crowdsourced user study that combines affectivepriming techniques from psychology with longstanding graphical perception experiments. Our results suggest that affective-priming can significantly influence accuracy in visual judgements, and that some chart types may be more affected than others.
\end{abstract}

\section{INTRODUCTION}

Effective analytical reasoning involves many cognitive subprocesses including memory, attention, learning, judgement, creativity, decision-making, and problem-solving. Interestingly, recent research in psychology has explored the extent to which emotion, even if short-lived, can significantly influence many of these abilities. For instance, performance on attention tasks are significantly impacted when emotion-inducing stimuli precedes the task [8].

In general, this technique of inducing emotion in human subjects is referred to as affective-priming. Based on research from psychology, we know that affective-priming influences cognitive and perceptual ability across various tasks. Coincidentally, in visualization and visual analytics, recent research has identified a close relationship between a user's cognitive abilities with the user's ability to perceive and understand information in visualizations [1] [3]. It appears that there could be a connection between the two. However, how affective-priming may influence a user's ability to perceive information in a visualization has not been a explored by researchers in visualization and visual analytics.

We note that there are many challenges for designing a controlled study for both affect and graphical perception individually, and even more so when combined. We handle these issues primarily by following previous graphical perception studies and using validated affective-priming techniques, as well as by increasing the scale of our study through crowdsourcing.

\section{EXPERIMENT}

To test how affect influences graphical perception, we conducted a large-scale experiment using Amazon's Mechanical Turk. We follow Heer-Bostock's crowdsourced replication of ClevelandMcGill's seminal study on graphical perception [2] [4], making necessary changes to effectively incorporate affective priming.

In a pilot study, we tested an initial hypothesis that charts that showed less error in Cleveland-McGill's study would be less influenced by affective-priming, and that charts ranked as more difficult would be more influenced. Based on this pilot, in which all charts were affected for successful primes in the intended direction (positive or negative), we formed the following hypothesis: Successful affective-priming will significantly influence error across all chart types.

*e-mail: 1tharri1@
Following previous affective-priming experiments [7], we evaluated several short stories found in the New York Times website for their emotional content. We chose two stories, one negative and one positive. These stories were validated as having the intended effect through an additional pilot study.

We chose the Self-Assessment Manikin (SAM) scale for testing the valence and arousal of the participant. The SAM scale is widely used in psychology, and is also well-suited for Mechanical Turk [6].

\subsection{Participants}

We recruited 963 participants through Amazon's Mechanical Turk system. It took a approximately 5 days to gather all responses. Our study used a total of 8 charts, and with the two priming groups (positive/negative) an average of 60 participants were assigned to each chart and priming type. After completing the study, participants were initially paid $\$ 0.20$, which was later increased to $\$ 0.35$ to speed completion time.

Participants were first given the SAM scale as a pre-test to record their valence and arousal. Next, participants were given the positive or negative story to read with the instructions that "the overall story is more important than the details". After reading was complete, participants were asked a simple verification question regarding the content of the story (the story was hidden at this point). Next, participants began the graphical perception task.

Each of the five charts (all being the same type: bar, pie, or one of the others) were presented in random order. Participants were instructed to make quick but accurate visual judgements, and to avoid using any outside tools. Similar to Heer-Bostock's study, participants were asked the following two questions about the charts: First, "Which of the two (A or B) is SMALLER?" followed by "What percentage is the SMALLER of the LARGER?".

After the graphical perception tasks were completed, participants were given the SAM again as a post-test. Finally, they were invited to submit any additional comments about the task.

\subsection{Results}

Our study adhered to a between-subjects design, since participants were given a single chart and priming combination. We excluded participants who incorrectly answered the story verification question, as well as those who either failed to answer or put the same answer for multiple questions (i.e. putting $50 \%$ for each judgement). In total, 299 of the 963 participants were removed. We note that the large amount of participants removed was due to the factors we used to ensure quality responses and to facilitate successful priming, mitigation techniques are discussed further in [4].

In our analysis we include those who were succesfully primed in the intended negative or positive direction. Specifically, we include those whose post-valence is higher than pre-valence for the positive group, and vice-versa for the negative group. Specifically, we include those whose post-valence is higher than pre-valence for the positive group, and vice-versa for the negative group. This resulted in 87 participants in the positive condition and 120 in the negative condition for a total of $n=197$. There was an average of 13 participants for a single chart+priming condition (out of 16 total possible combinations). As stated before, each participant made judgements on 5 charts. Therefore, this analysis consists of approximately 985 individual judgements. These results are shown in figure 2 . 


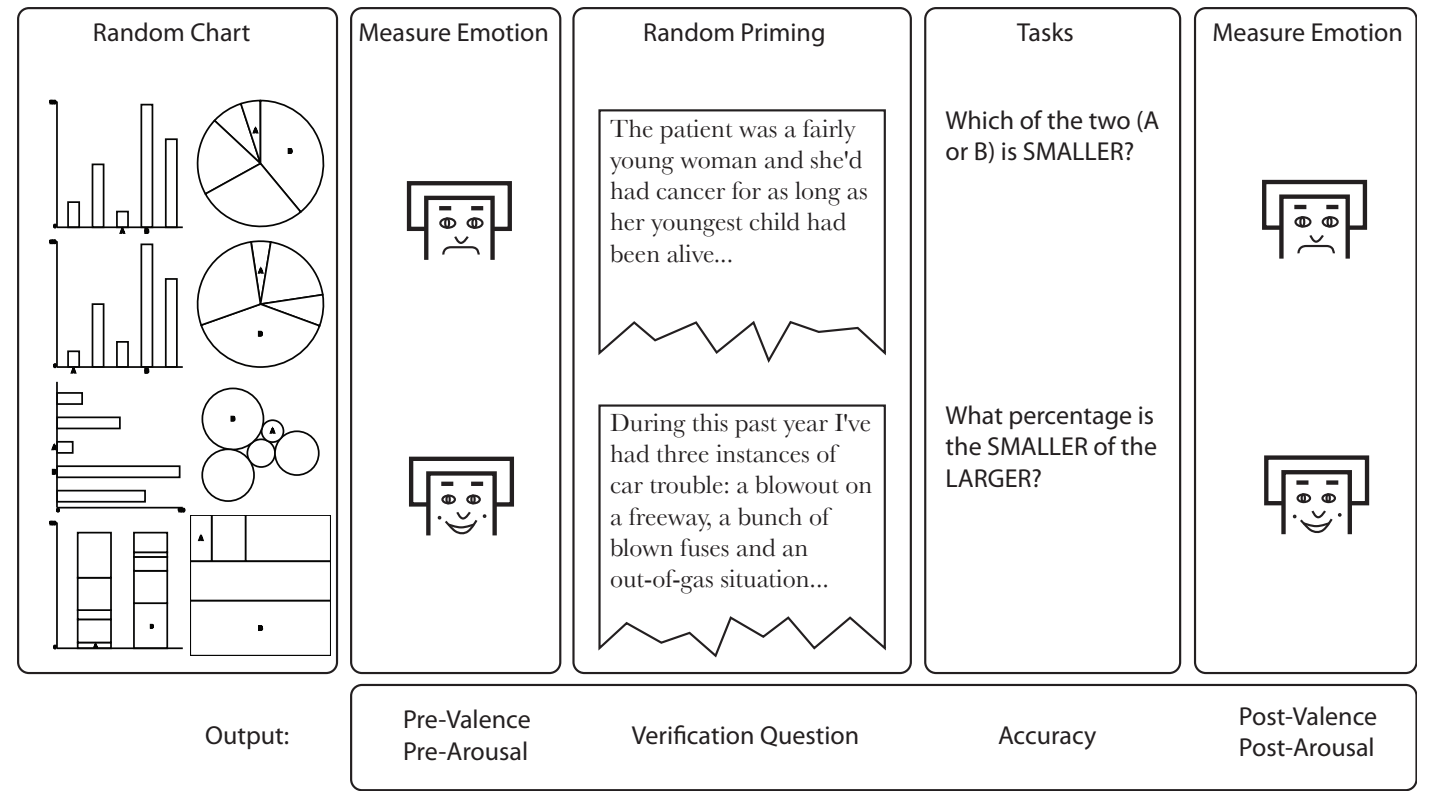

Figure 1: Experiment design: each participant is randomly assigned chart type, which is then used in the series of judgement tasks.

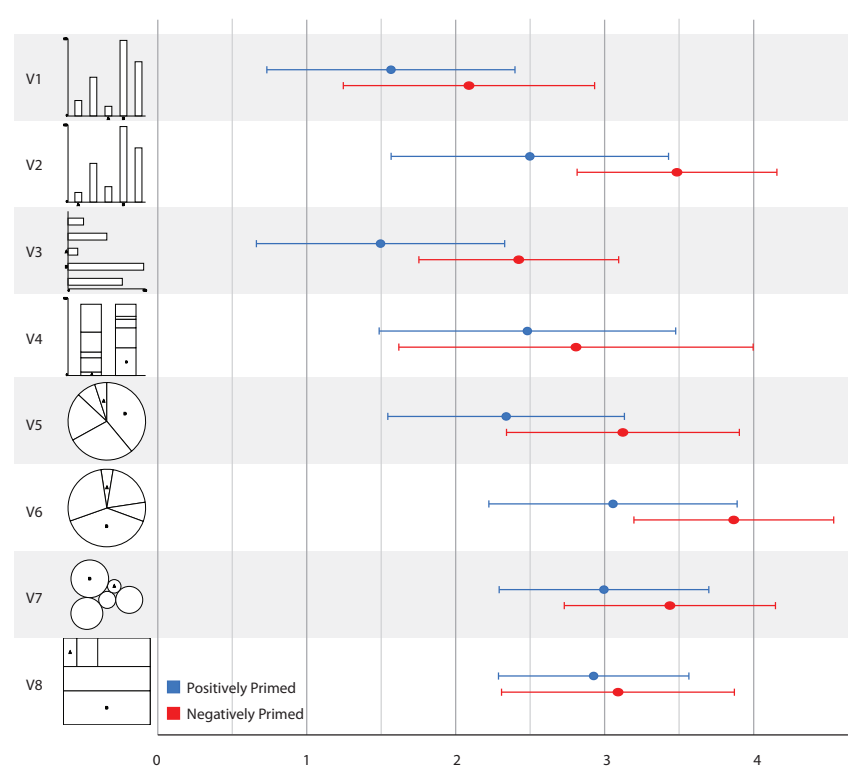

Figure 2: Error for successfully primed participants.

Following Hullman et al., we also take the results for all charts in each priming group together, including all chart error scores for each participant in the negative- and positive- primed groups [5]. We then compare positive error with negative error using a t-test. This yields a signficant effect for error $t(205)=3.1560, p=.0018$, with error in the negative group being higher than that of the positive group. The lowest error appeared in the positive group $(\mathrm{M}=$ $2.42, \mathrm{SD}=1.47$ ), meaning participants in the positive group performed better on graphical perception tasks than those in the negative group $(\mathrm{M}=3.05, \mathrm{SD}=1.49)$ (see figure 3 ). These results are consistent with our hypothesis that affective-priming can significantly impact graphical perception performance in participants who report delta in valence scores.

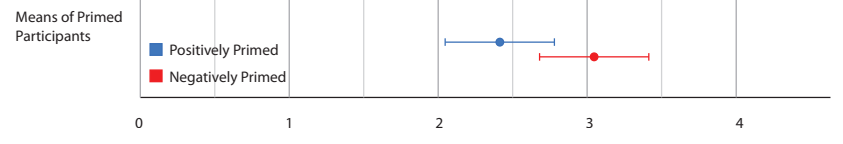

Figure 3: Error of combined means (negative, positive) for successfully primed participants.

\section{Conclusion}

The main finding of this work is that affective priming can influence graphical perception. This was accomplished through a large-scale crowdsourced study. Our results suggest that there are significant differences in graphical perception ability for positive and negative affective-primes, and that positive priming tends to increase graphical perception ability, rather than negative priming decreasing ability. We hope these findings serve as a step forward in better understanding the cognitive aspects related to visualization.

\section{REFERENCES}

[1] C. Chen and M. Czerwinski. Spatial ability and visual navigation: An empirical study. New Review of Hypermedia and Multimedia, 1997.

[2] W. S. Cleveland and R. McGill. Graphical perception: Theory, experimentation, and application to the development of graphical methods. Journal of the American Statistical Association, 1984.

[3] C. Conati and H. Maclaren. Exploring the role of individual differences in information visualization. In Proc. of the working conference on advanced visual interfaces. ACM, 2008.

[4] J. Heer and M. Bostock. Crowdsourcing graphical perception: Using mechanical turk to assess visualization design. Human factors in computing systems (CHI), 2010.

[5] J. Hullman, E. Adar, and P. Shah. The impact of social information on visual judgments. Human factors in computing systems (CHI), 2011.

[6] S. Lewis, M. Dontcheva, and E. Gerber. Affective computational priming and creativity. Human factors in computing systems (CHI), 2011.

[7] C. Verheyen and A. Goeritz. Plain texts as an online mood-induction procedure. Social Psychology, 2009.

[8] P. Vuilleumier and J. Driver. Modulation of visual processing by attention and emotion: Windows on causal interactions between human brain regions. Philosophical Transactions: Biological Sciences, 2007. 